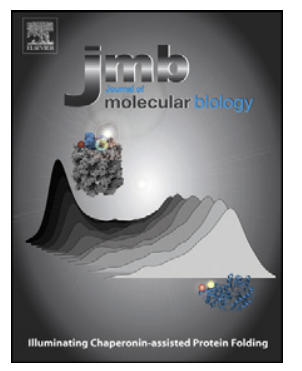

\title{
Active Cage Mechanism of Chaperonin-Assisted Protein Folding Demonstrated at Single-Molecule Level
}

\author{
Amit J. Gupta ${ }^{\dagger}$, Shubhasis Haldar ${ }^{\dagger}$, Goran Miličić, \\ F. Ulrich Hartl and Manajit Hayer-Hartl
}

Department of Cellular Biochemistry, Max Planck Institute of Biochemistry, Am Klopferspitz 18, 82152 Martinsried, Germany

\section{Correspondence to}

F. Ulrich Hartl and Manajit Hayer-Hartl:

uhartl@biochem.mpg.de; mhartl@biochem.mpg.de

http://dx.doi.org/10.1016/j.jmb.2014.04.018

Edited by R. L. Gonzalez

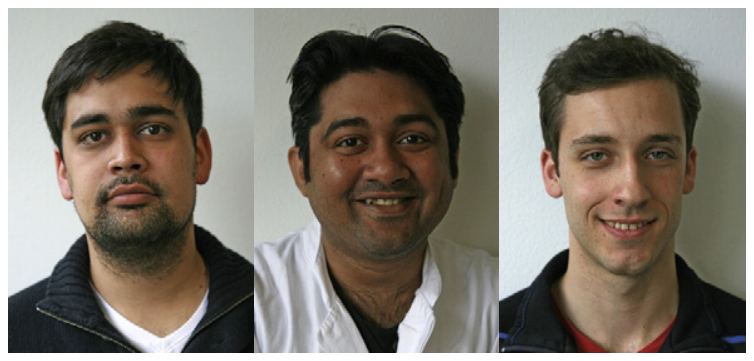

Amit J. Gupta, Shubhasis Haldar and Goran Miličić

\section{Abstract}

The cylindrical chaperonin GroEL and its lid-shaped cofactor GroES of Escherichia coli have an essential role in assisting protein folding by transiently encapsulating non-native substrate in an ATP-regulated mechanism. It remains controversial whether the chaperonin system functions solely as an infinite dilution chamber, preventing off-pathway aggregation, or actively enhances folding kinetics by modulating the folding energy landscape. Here we developed single-molecule approaches to distinguish between passive and active chaperonin mechanisms. Using low protein concentrations (100 pM) to exclude aggregation, we measured the spontaneous and GroEL/ES-assisted folding of double-mutant maltose binding protein (DM-MBP) by single-pair fluorescence resonance energy transfer and fluorescence correlation spectroscopy. We find that GroEL/ES accelerates folding of DM-MBP up to 8-fold over the spontaneous folding rate. Accelerated folding is achieved by encapsulation of folding intermediate in the GroEL/ES cage, independent of repetitive cycles of protein binding and release from GroEL. Moreover, photoinduced electron transfer experiments provided direct physical evidence that the confining environment of the chaperonin restricts polypeptide chain dynamics. This effect is mediated by the net-negatively charged wall of the GroEL/ES cavity, as shown using the GroEL mutant EL(KKK2) in which the net-negative charge is removed. EL(KKK2)/ES functions as a

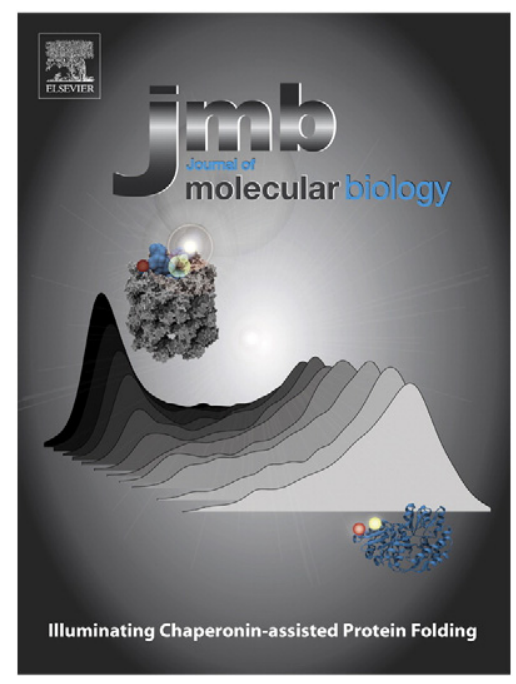

Legend. The GroEL/ES-mediated folding of the model substrate protein DM-MBP is monitored by single-molecule FRET. Folding intermediate bound to GroEL shows a Iow FRET efficiency distribution. Upon refolding, DM-MBP converts to its compact native structure that exhibits a high FRET efficiency distribution.

passive cage in which folding occurs at the slow spontaneous rate. Taken together our findings suggest that protein encapsulation can accelerate folding by entropically destabilizing folding intermediates, in strong support of an active chaperonin 
mechanism in the folding of some proteins. Accelerated folding is biologically significant as it adjusts folding rates relative to the speed of protein synthesis.

(C) 2014 The Authors. Published by Elsevier Ltd. This is an open access article under the CC BY-NC-ND license (http://creativecommons.org/licenses/by/3.0/).

\section{Introduction}

Chaperonins are large ATP-driven macromolecular machines composed of two rings of $\sim 60 \mathrm{kDa}$ subunits stacked back to back. They have an essential role in assisting protein folding in bacteria, archaea and eukarya [1-6]. Group I chaperonins occur in bacteria (GroEL), mitochondria (Hsp60) and chloroplasts (Cpn60). They consist of heptameric rings and functionally cooperate with lid-like cofactors (GroES in bacteria, Hsp10 in mitochondria and Cpn10/Cpn20 in chloroplasts) that function to transiently encapsulate non-native substrate protein in a cage-like compartment. Group II chaperonins in archaea and the cytosol of eukaryotic cells have rings of 8-9 subunits and employ a mechanism of opening and closing their central cavity that is in-built into the structure of the chaperonin ring.

The group I chaperonins GroEL and GroES of Escherichia coli have been investigated most widely and numerous biochemical and structural studies indicate that they function as a nano-compartment for single protein molecules to fold in isolation [7-23]. However, whether protein encapsulation actively promotes folding remains controversial $[12,17,19,22,24-27]$.

Each subunit of GroEL is divided into apical, intermediate and equatorial domains [28] (Fig. 1a). The apical domains form the ring opening and expose hydrophobic amino acid residues for the binding of molten globule-like folding intermediates [29-31]. ATP binding and hydrolysis in the equatorial domains results in conformational changes that are transduced to the apical domains via the hinge-like intermediate domains, regulating substrate affinity and GroES binding through an allosteric reaction cycle $[4,32]$. In the current model, non-native protein substrate binds to the open ring (trans-ring) of an asymmetrical GroEL/ ES complex (Fig. 1b) (The corresponding video file for the animated Abstract can be found online at doi:10.1016/j.jmb.2014.04.018). Subsequent ATP binding causes apical domain movements that may result in stretching the bound polypeptide [18,33-36], and at the same time, ADP and GroES dissociate from the opposite ring (Fig. 1b). ATP binding is closely followed by GroES binding, resulting in the displacement of the bound substrate and its encapsulation in the newly formed GroEL/ES cage (cis-ring). Encap- sulated protein (up to $\sim 60 \mathrm{kDa}$ in size) is now free to fold unimpaired by aggregation in a cage with a hydrophilic, net-negatively charged wall (in cagefolding). The time allowed for folding is dependent on the rate of hydrolysis of the seven ATP in the cis-ring $\left(\sim 5-10 \mathrm{~s}\right.$ at $\left.25^{\circ} \mathrm{C}\right)$. After completion of ATP hydrolysis, ATP binding to the GroEL trans-ring causes dissociation of ADP and GroES (Fig. 1b). Folded protein is released, while not-yet folded protein will be rapidly recaptured for possible stretching, encapsulation and folding. Symmetrical GroEL/ES complexes with GroES bound to both GroEL rings have also been observed, but their function in the reaction cycle is still under investigation $[27,37,38]$.

Three models have been proposed to explain how this basic chaperonin cycle promotes protein folding. The "passive cage" (also known as "Anfinsen cage") model suggests that GroEL/ES essentially provides an infinite dilution chamber $[25,39,40]$. The rate of spontaneous folding, when measured in the absence of reversible aggregation, is identical with the rate of folding inside the cage. The model implies that GroEL/ES-dependent proteins fold at a biologically relevant timescale as long as aggregation is prevented. In contrast, the "active cage" model states that, besides preventing aggregation, the physical environment of the cage also modulates the folding energy landscape, resulting in accelerated folding of certain proteins. This is attributed to an effect of steric confinement that limits the conformational space to be explored during folding $[12,17,19,22,41-43]$. The active cage model implies that cells contain a set of proteins with kinetically frustrated folding pathways that require folding catalysis to reach their native states at a biologically relevant speed. Finally, the "iterative annealing" model posits that the function of GroEL/ES is to unfold misfolded proteins through cycles of binding and release, with folding occurring either inside or outside the cage $[27,44]$ (out of cage-folding; Fig. 1b). In this model, accelerated folding may result from the active unfolding of kinetically trapped species that can then partition between productive and unproductive folding trajectories. The transient encapsulation of substrate is thought to be a mere by-product of the unfolding reaction [27].

In an effort to distinguish between these models, we developed novel approaches to investigate protein folding by GroEL/ES at single-molecule level. Using single-pair fluorescence resonance energy transfer (spFRET), dual-color fluorescence cross-correlation spectroscopy (dcFCCS) and photoinduced electron transfer (PET), we could exclude reversible aggregation as the cause of slow spontaneous folding and unequivocally distinguish between active and passive chaperonin mechanisms. We find that GroEL/ES accelerates the folding of a double-mutant maltose binding protein (DM-MBP) up to $\sim 8$-fold relative to its 
a GroEL subunit GroEL
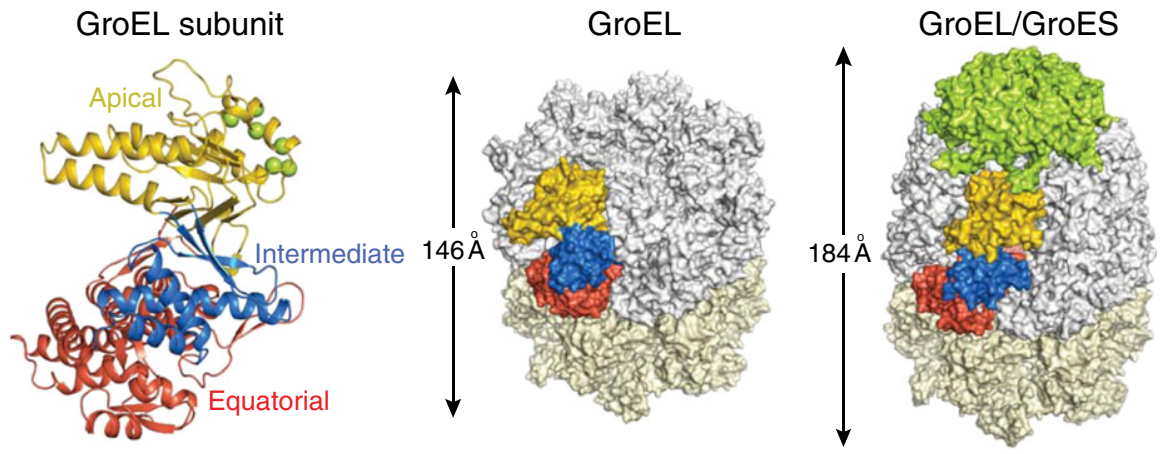

b

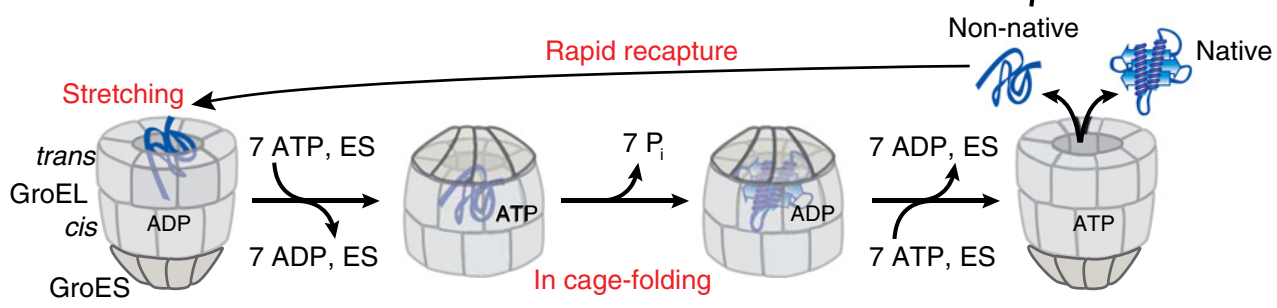

Fig. 1. Structure and function of the GroEL/ES chaperonin. (a) Left: Structure of the GroEL subunit in the apo-state in ribbon representation (PDB ID 1SS8). Apical domain, yellow; intermediate domain, blue; equatorial domain, red. Middle: Structure of the apo-GroEL 14-mer (PDB ID 1SS8). One subunit of GroEL is shown in color. Right: Structure of the GroEL/ GroES complex in the ADP state (PDB ID 1PF9). The two rings of GroEL are shown in gray and beige, and GroES is shown in green. (b) Model of the GroEL/GroES reaction cycle. See Introduction and Discussion for details.

spontaneous rate. Accelerated folding occurs upon a single round of encapsulation, as demonstrated using SREL, a single-ring variant of GroEL that results in stable protein encapsulation without GroES dissociation. Thus, multiple rounds of substrate binding and release as proposed by the iterative annealing model are not required for folding catalysis. Instead, accelerated folding is due to the physical confinement of non-native protein in the net-negatively charged GroEL/ES cage. Consistent with this mechanism, we demonstrate that, during the GroEL/ES reaction cycle, the substrate protein spends $\sim 82 \%$ of its time inside the chaperonin cage and only $\sim 18 \%$ in the GroEL-bound state, with negligible amounts of non-native protein being free in solution.

\section{Results}

\section{Transient aggregation is not the cause of slow spontaneous folding}

We used DM-MBP ( 41 kDa), a double mutant of MBP, which has previously been used as a model substrate to compare the rates of spontaneous and GroEL/ES-assisted folding [17,22,45]. DM-MBP carries mutations V8G and Y283D that delay the rate-limiting folding step of the $\mathrm{N}$-domain [46] (Fig. 2a). As a result, the spontaneous refolding of DM-MBP is slow $\left(t_{1 / 2} \sim 35 \mathrm{~min}\right.$ at $25^{\circ} \mathrm{C}$ and physiological salt concentration) but nevertheless fully efficient [17,24], with only largely unstructured intermediate and the native state being populated during folding [22]. The GroEL/ES-assisted folding of DM-MBP has a 6- to 10-fold faster rate. However, there is disagreement whether the observed rate acceleration is due to GroEL/ES actively modulating the folding energy landscape [17] or to GroEL/ES passively preventing reversible aggregation that would slow spontaneous folding $[24,26]$.

To establish conditions of spontaneous refolding in which transient aggregation is excluded, we resorted to single-molecule fluorescence measurements. A single cysteine mutant of DM-MBP, DM-MBP(312C), was labeled with the fluorophore Atto532 or Atto647N and used in dual-color fluorescence cross-correlation (dcFCCS) experiments. The native, labeled proteins were mixed $1: 1$ at a final concentration of $50 \mathrm{pM}$ each. The probability, at $100 \mathrm{pM}$, for two monomeric DM-MBP molecules to be simultaneously present in the observation volume $(1 \mathrm{fL})$ is $\leq 1 \%$. As expected, no cross-correlation 
a

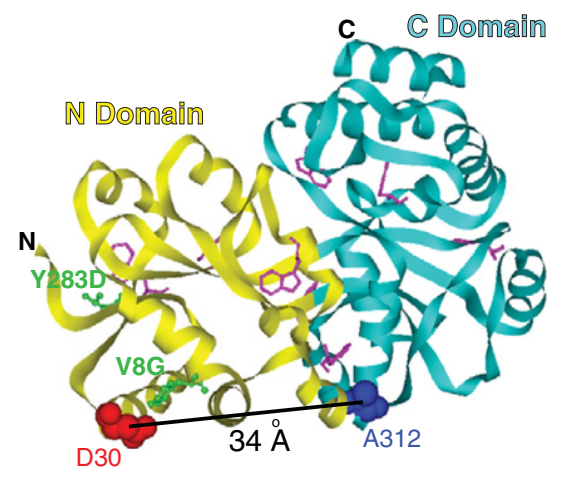

b

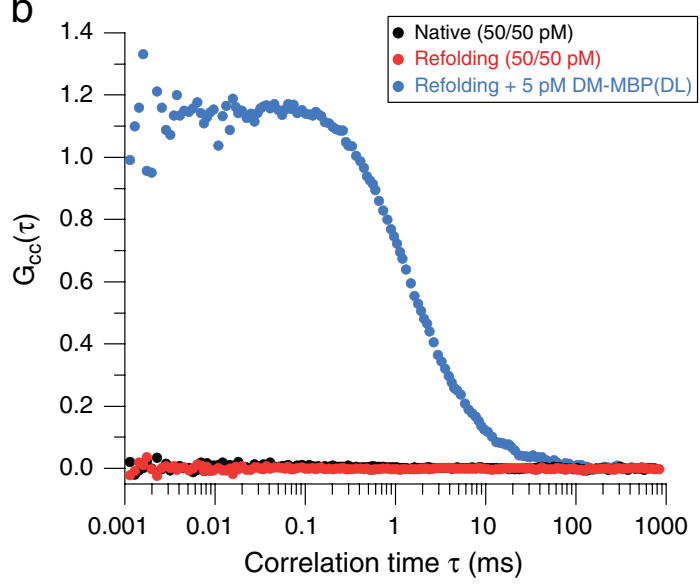

C

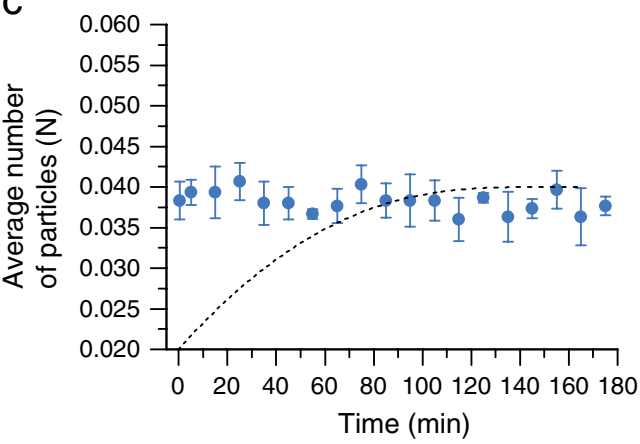

Fig. 2. Absence of DM-MBP aggregation during spontaneous refolding. (a) Ribbon structure of maltose binding protein (MBP) (PDB ID 1OMP). The discontinuous N-and C-domains are shown in yellow and blue, respectively. The positions of mutations Y283D and V8G (green) in DM-MBP, the 8 Trp residues (pink) and the cysteine substitutions at D30 and A312 used for fluorescence labeling are indicated. (b) Absence of FCCS signal $G_{\mathrm{CC}}(\mathrm{T})$ during spontaneous refolding of DM-MBP. A 1:1 mixture of DM-MBP(312C) labeled with either Atto532 or Atto647N was denatured in $6 \mathrm{M} \mathrm{GuHCl}$ and $10 \mathrm{mM}$ DTT for $1 \mathrm{~h}$ at $20^{\circ} \mathrm{C}$. Refolding was initiated by 200 -fold dilution into buffer $\mathrm{A}$ to a final concentration of $50 \mathrm{pM}$ each. dcFCCS was recorded with pulsed interleaved excitation [60] within the first $10 \mathrm{~min}$ of refolding (red). As a positive control, $5 \mathrm{pM}$ native, double-labeled protein, $\mathrm{DM}-\mathrm{MBP}(\mathrm{DL})$, was added to the mixture of single-labeled, denatured proteins during refolding to simulate the presence of an oligomeric (dimeric) species (blue). A 1:1 mixture of the native, single-labeled proteins (50 pM each) was analyzed as a negative control (black). Shown are representative results of least three independent experiments. (c) Average number of fluorescent-labeled particles inside the confocal observation volume during the course of spontaneous refolding of $100 \mathrm{pM}$ DM-MBP(312C) labeled with Atto647N in buffer A. FCS was recorded over the course of $3 \mathrm{~h}$. FCS analysis was performed for continuous time windows of $10 \mathrm{~min}$ and the average number of particles was extracted from the amplitude of the fit. Averages \pm SD from three independent experiments are shown. Dotted line is the simulated increase in number of particles assuming that reversible aggregation limits spontaneous refolding.

signal was observed (Fig. 2b). To investigate DM-MBP under refolding conditions, we denatured the differently labeled DM-MBP molecules in $\mathrm{GuHCl}$ as a 1:1 mixture and allowed them to refold upon dilution from denaturant at $100 \mathrm{pM}$ final concentration. No cross-correlation signal was observed during refolding (Fig. 2b). The sensitivity of the method was demonstrated using the double cysteine mutant DM-MBP(30C/312C) labeled with Atto532 and Atto647N [DM-MBP(DL)]. A strong cross-correlation signal was observed when $5 \mathrm{pM}$ of the double-labeled protein, mimicking the presence of dimeric aggregates, was added to the $100 \mathrm{pM}$ mixture of the single-labeled refolding proteins (Fig. 1b). These measurements show clearly that, at $100 \mathrm{pM}$, the labeled DM$\mathrm{MBP}(312 \mathrm{C})$ proteins are monomeric during refolding and do not form oligomers.

Analysis by fluorescence correlation spectroscopy (FCS) further confirmed the absence of reversible aggregates by demonstrating that the number of Atto647N-labeled DM-MBP(312C) particles in the observation volume remained constant over the refolding time (Fig. 2c). In contrast, if reversible aggregation was to limit the rate of spontaneous folding, the number of diffusing particles would be expected to increase as native monomeric protein is produced (Fig. 2c, simulated broken curve). 


\section{Spontaneous and GroEL/ES-assisted folding measured at single-molecule level}

Having established conditions of spontaneous refolding in the absence of aggregation, we next developed a spFRET approach to measure the rates of spontaneous and GroEL/ES-assisted refolding at single-molecule level. Specifically, we tested the prediction of the passive cage model that, under conditions equivalent to infinite dilution, no rate acceleration by chaperonin would be observed [24]. As shown previously, DM-MBP(DL) in its native state and the unfolded protein when bound to GroEL have different FRET efficiency $\left(f_{\mathrm{E}}\right)$ distributions in single-molecule spFRET measurements [18]. The native protein shows a distribution of compact conformations with a peak at a high $f_{\mathrm{E}}$ of 0.72 (Fig. 3a). In contrast, GroEL-bound DM-MBP(DL) has $\sim 40 \%$ molecules at a low $f_{E}$ of 0.06 , consistent with a highly expanded conformation, with the remainder of molecules populating a broad distribution of less expanded states around an intermediate $f_{\mathrm{E}}$ of 0.38 (Fig. 3b). To obtain the kinetics of spontaneous folding, we took advantage of the ability of GroEL to bind folding intermediates, but not the native protein, thereby stopping refolding and reverting not-yet folded DM-MBP(DL) molecules to the low $f_{\mathrm{E}}$ distribution (stretching; Fig. 1b). Assisted folding in the presence of GroEL/ES and ATP was stopped by the addition of apyrase, resulting in rapid hydrolysis of ATP and ADP to AMP. Quantification of the low and high $f_{\mathrm{E}}$ peak areas $\left(f_{\mathrm{E}}\right.$ of 0.06 and 0.72 , respectively) at different times enabled us to extract protein folding rates at a concentration of $100 \mathrm{pM}$ DM-MBP(DL) (Fig. 3c and d). The rate of spontaneous refolding was $\sim 5.6$-fold slower than the assisted rate (Fig. 3e). To validate our findings, we measured the refolding rate of $\mathrm{DM}-\mathrm{MBP}(\mathrm{DL})$ at a protein concentration of $100 \mathrm{nM}$. In these ensemble experiments we took advantage of the finding that, following initial collapse (with decrease in donor fluorescence due to FRET), the donor fluorescence of DM-MBP(DL) increases during folding, apparently due to changes in the chemical environment of the fluorophore. The observed rates were in good agreement with the single-molecule data and showed a 7.7-fold acceleration of folding by chaperonin (Fig. 3f). Moreover, similar folding rates were previously measured for the unlabeled DM-MBP(30C/312C) by tryptophan fluorescence [18].

As an alternative single-molecule approach to measure folding rates, we utilized the difference in diffusion coefficients $(D)$ of GroEL bound $\left(\sim 49 \mu \mathrm{m}^{2} \mathrm{~s}^{-1}\right)$ and free DM-MBP $\left(\sim 160 \mu \mathrm{m}^{2} \mathrm{~s}^{-1}\right)$ by FCS (Fig. 4a). We recorded the time-dependent change in the average diffusion rate during refolding of $100 \mathrm{pM}$ DM-MBP(DL) using the Atto647N fluorescence signal (Fig. S1). Again, spontaneous folding was stopped by addition of excess GroEL and the assisted folding with apyrase. The folding rates obtained in these measurements (Fig. 4b) were in agreement with those obtained by spFRET (Fig. 3e).

The previously reported effect of chloride salt to slow the spontaneous refolding of DM-MBP $[22,26]$ was preserved under single-molecule conditions where aggregation is excluded (Fig. S2). Consequently, chloride salt does not decelerate spontaneous refolding by increasing aggregation [26] but by modulating the intrinsic folding properties of DM-MBP [22]. The electrostatic environment of the GroEL/ES cage apparently renders DM-MBP refolding salt insensitive [22].

\section{PET-FCS as a measure of chain motion during folding}

The active cage model of chaperonin action posits that encapsulation of non-native protein can reduce chain entropy, thereby accelerating folding kinetics $[12,17,22]$. Here we used fluorescence quenching via PET to test this hypothesis. In PET, the fluorescence of an oxazine dye (Atto655) is quenched via van der Waals contact with a Trp residue by direct transfer of an electron. Atto655 has the advantage of showing virtually no triplet blinking or other photophysical fluctuations $[47,48]$ and thus is well suited to assess conformationally induced fluctuations at fast timescales. MBP contains 8 Trp residues spaced throughout the sequence (Fig. 2a) (note that GroEL and GroES do not contain Trp). The combination of PET with FCS serves as a powerful method to measure structural fluctuations in proteins at the single-molecule level on timescales from nanoseconds to milliseconds [48]. PET-FCS has been used to study denatured state dynamics and early events in protein folding [47].

The auto-correlation signal of Atto655-labeled DM-MBP(312C) [DM-MBP(Atto655)] was measured $30 \mathrm{~s}$ after dilution from denaturant, when essentially all DM-MBP populates a dynamic folding intermediate that converts only slowly to the native state [22]. The auto-correlation curve could not be fitted with a single component diffusion model due to the presence of a fast fluctuating component (Fig. 5a). It was fitted by a one-exponential one-diffusion equation (Fig. 5b), with the exponential term describing the PET amplitude $F$, which is proportional to the abundance of conformationally dynamic molecules, and the relaxation time $\left(T_{R}\right)$ providing a measure of chain motion (Fig. S3a). Based on these measurements, the slow folding intermediate of DM-MBP(Atto655) shows fast fluctuations of the fluorescence signal at a $T_{R}$ of $40 \pm 3 \mu \mathrm{s}$, indicating high chain entropy. As a control, Atto655-labeled wild-type $\operatorname{MBP}(312 \mathrm{C})$ showed no fast fluorescence fluctuation during folding (Fig. $5 \mathrm{c}$ ), as this rapidly folding protein $\left(t_{1 / 2} \sim 23 \mathrm{~s}\right)$ [17] does not significantly 
populate the dynamic intermediate in which the dye can approach Trp residues. Similarly, when DM-MBP(Atto655) was allowed to refold to completion, the fluorescence fluctuations at the short correlation times were no longer detected (Fig. 5d). This is consistent with the fact that no Trp residue is in contact distance to residue 312 in the native state. Thus, fast fluorescence fluctuation is characteristic of the dynamic folding intermediate of DM-MBP.

The amplitude $F$ of the PET-FCS signal (Fig. S3a), reflecting the fraction of conformationally dynamic molecules, decreased during spontaneous refolding (Fig. 5e) at a rate equivalent to that measured for folding of the unlabeled protein by Trp fluorescence
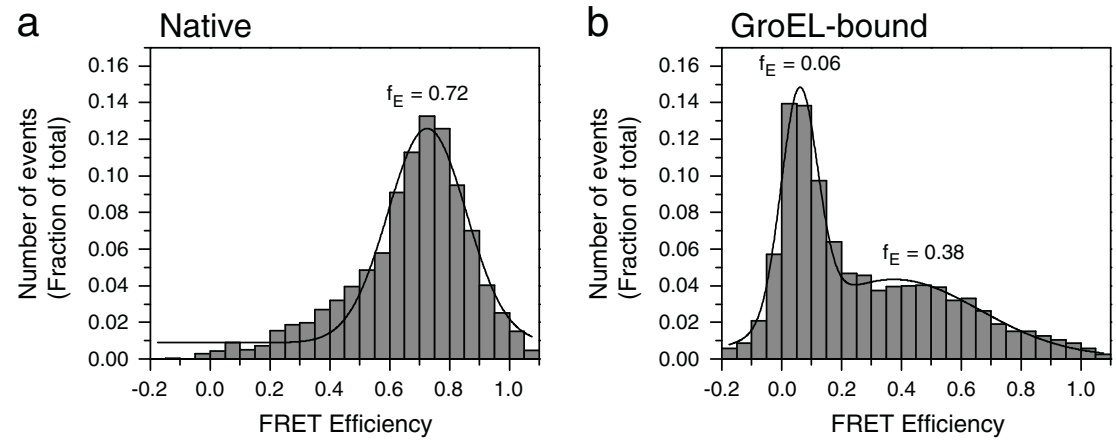

C Spontaneous refolding
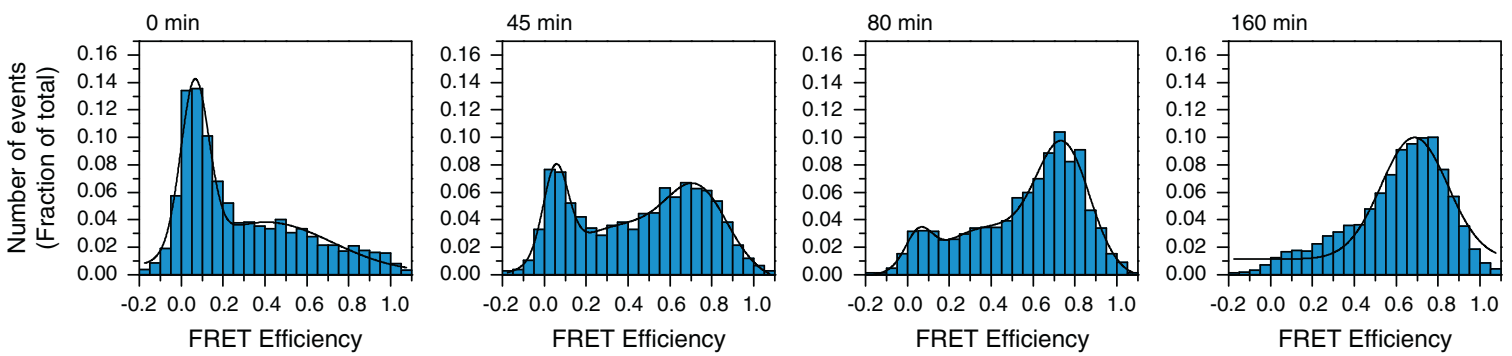

d GroEL/ES-assisted
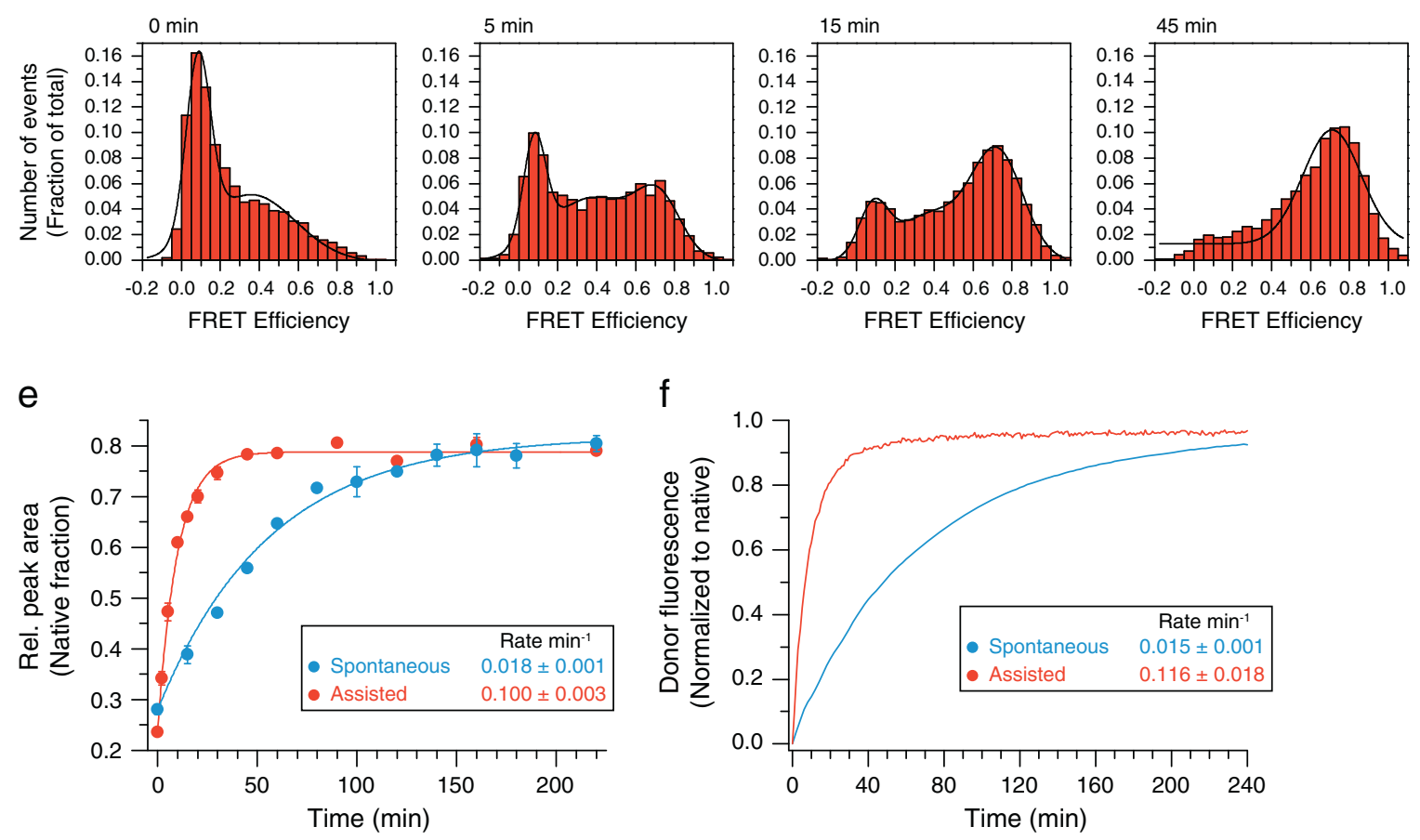
a

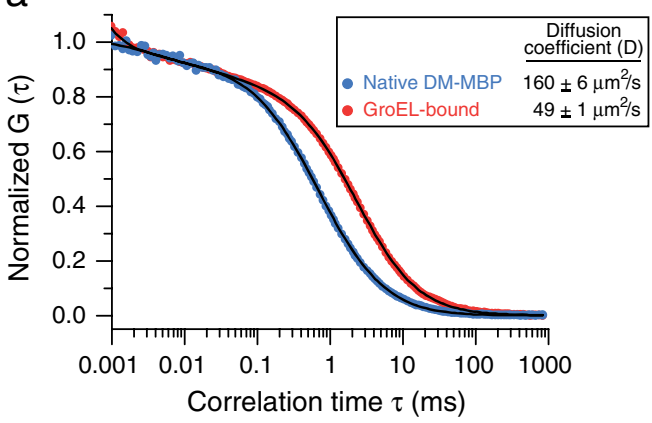

b

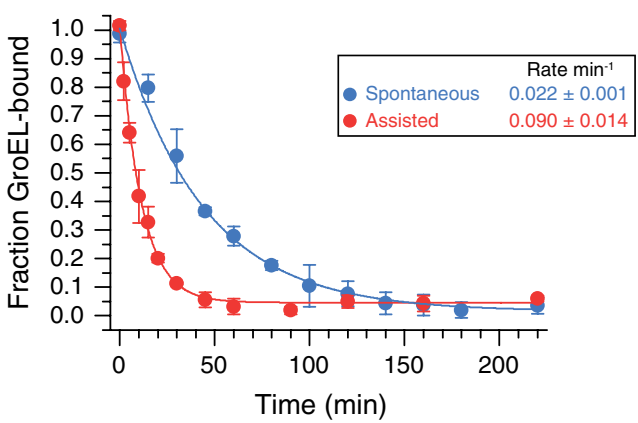

Fig. 4. Spontaneous and GroEL/ES-assisted refolding of DM-MBP measured by FCS. (a) Normalized fluorescence auto-correlation amplitudes $G(\mathrm{~T})$ of Atto647N fluorescence for $100 \mathrm{pM}$ GroEL bound and spontaneously refolded DM-MBP(DL) in buffer A. Diffusion coefficients $(D)$ are indicated. \pm SD from at least 3 independent measurements. (b) Refolding kinetics based on the difference in mean diffusion time through the confocal volume between GroEL-bound and free, native protein. Refolding was performed as in Fig. 3 and stopped either by adding GroEL (spontaneous refolding) or apyrase (assisted refolding), shifting not-yet folded DM-MBP to the slow diffusion time of the GroEL complex. Refolding is plotted as fraction of GroEL-bound protein and fitted to single exponential rates. \pm SD from at least 3 independent measurements.
(Fig. S3b). This would be expected if refolding is limited by a kinetic energy barrier with a large entropic component. The rate of decrease in amplitude $F$ was accelerated $\sim$ 4-fold during GroEL/ES-assisted refolding (Fig. 5e), consistent with the chaperonin system reducing the entropic component of the energy barrier. In contrast, a constant high PET-FCS signal was observed when the labeled protein was diluted into buffer containing $0.5 \mathrm{M} \mathrm{GuHCl}$, which stabilizes DM-MBP in its dynamic intermediate state [22] (Fig. 5e). The rate of spontaneous refolding of DM-MBP(Atto655) measured by PET-FCS was concentration independent over a concentration range of 4 orders of magnitude (100 pM to $1 \mu \mathrm{M}$ ) (Fig. S3c), further excluding aggregation as the cause of slow spontaneous refolding.

\section{Evidence for conformational confinement in the GroEL/ES cage}

While the amplitude of the PET-FCS signal is proportional to the concentration of dynamic particles, the $T_{R}$ of the PET signal is indicative of the kinetics of chain motion. During the first minute of spontaneous refolding, the $T_{R}$ of DM-MBP(Atto655) was $40 \pm 3 \mu \mathrm{s}$, similar to the $T_{R}$ measured for the kinetically trapped intermediate in $0.5 \mathrm{M} \mathrm{GuHCl}$ $(34 \pm 10 \mu \mathrm{s})$ (Fig. 5f). Note that no $T_{R}$ could be measured for the native protein (Fig. $5 \mathrm{~d}$ ). The $T_{R}$ of the GroEL-bound protein was $59 \pm 10 \mu \mathrm{s}$, indicating that the interaction of the unfolded DM-MBP with the apical GroEL domains reduces chain dynamics (Fig. 5f). Interestingly, the $T_{R}$ during the first minute of GroEL/ES-assisted refolding ( $\leq 20 \%$ molecules folded) was increased to $96 \pm 5 \mu$ s (Fig. 5f), suggesting that the encapsulated protein is significantly restricted in chain motion, even when compared to the GroEL-bound state. To test this further, we measured chain mobility upon stable encapsulation of unfolded DM-MBP in the non-cycling SREL/ES complex (Fig. 5f). SREL is a single-ring variant of

Fig. 3. Spontaneous and GroEL/ES-assisted refolding of DM-MBP measured by spFRET. (a and b) FRET efficiency $\left(f_{E}\right)$ histograms from spFRET measurements of native (a) and GroEL-bound (b) DM-MBP(DL) at 100 pM concentration (GroEL, $2 \mu \mathrm{M})$. (c) $f_{\mathrm{E}}$ histograms upon spontaneous refolding of $100 \mathrm{pM}$ DM-MBP(DL). Refolding was performed in buffer A and stopped at the times indicated by addition of $2 \mu \mathrm{M}$ GroEL, followed by spFRET analysis for $1 \mathrm{~h}$. Peak values of a Gaussian fit to the $f_{\mathrm{E}}$ distributions are indicated. Representative histograms for the refolding times of $0,45,80$ and 160 min are shown. (d) $f_{\mathrm{E}}$ histograms upon GroEL/ES-assisted refolding of $100 \mathrm{pM}$ DM-MBP(DL). Unfolded protein was $200-\mathrm{fold}$ diluted from $6 \mathrm{M} \mathrm{GuHCl}$ into buffer A containing $2 \mu \mathrm{M}$ GroEL. Refolding was initiated by addition of $4 \mu \mathrm{M}$ GroES/5 mM ATP and stopped by addition of apyrase at the times indicated. Representative histograms for the assisted refolding times of 0 , 5, 15 and 45 min are shown. Histograms shown in (a-d) are representative of least three independent experiments. (e) Kinetics for spontaneous and assisted refolding were obtained from spFRET measurements as in (c) and (d) by plotting the time-dependent increase of the area of the high $f_{\mathrm{E}}$ peak, corresponding to native DM-MBP(DL). Data were fitted with a single exponential rate. Data represent averages \pm SD from at least 3 independent measurements. (f) Refolding kinetics of $100 \mathrm{nM}$ DM-MBP(DL) measured by conventional fluorescence spectroscopy in an ensemble approach. Refolding curves in buffer A were monitored over time at donor excitation and emission wavelengths of $532 \mathrm{~nm}$ and $550 \mathrm{~nm}$, respectively, and are plotted as donor fluorescence relative to native. Averages \pm SD from at least 3 independent measurements are shown. 
GroEL that binds unfolded protein, ATP and GroES but ceases to hydrolyze ATP after a single round due to the absence of the allosteric signal from the GroEL trans-ring (see Fig. 1b) [8]. The SREL/ES complex is salt sensitive $[9,49]$, and thus, the experiments with SREL were performed in low salt buffer using urea-denatured DM-MBP(Atto655). Stable substrate encapsulation was confirmed by size-exclusion chromatography (Fig. S4). Approximately 90-95\% of DM-MBP co-fractionated with SREL/ES during folding, with the remainder eluting at a low molecular weight corresponding to free DM-MBP (Fig. S4a and b). Refolded DM-MBP was retained in the SREL/ES complex for more than 30 min (Fig. S4b, top panel) but was rapidly released when the SREL/ES complex was dissociated by addition of $\mathrm{Mg}$ chelator and high salt (Fig. S4b, bottom panel). The $T_{R}$ during the first minute of encapsulation in SREL/ES-assisted refolding was $99 \pm 1 \mu \mathrm{s}$, identical with the value measured with the cycling GroEL/ES system under the same low a

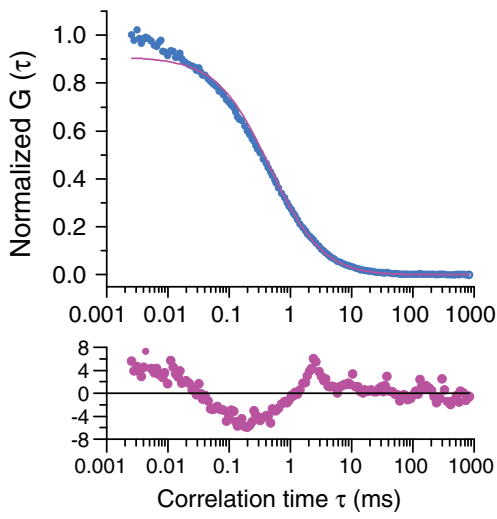

d

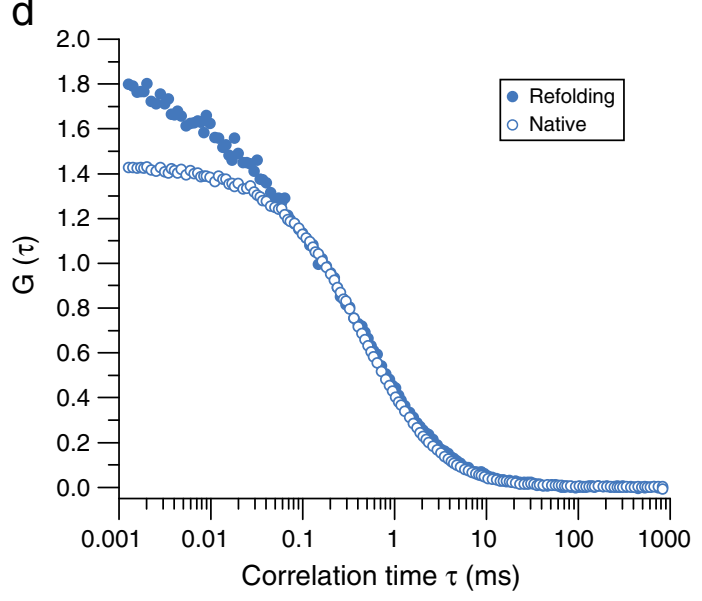

f

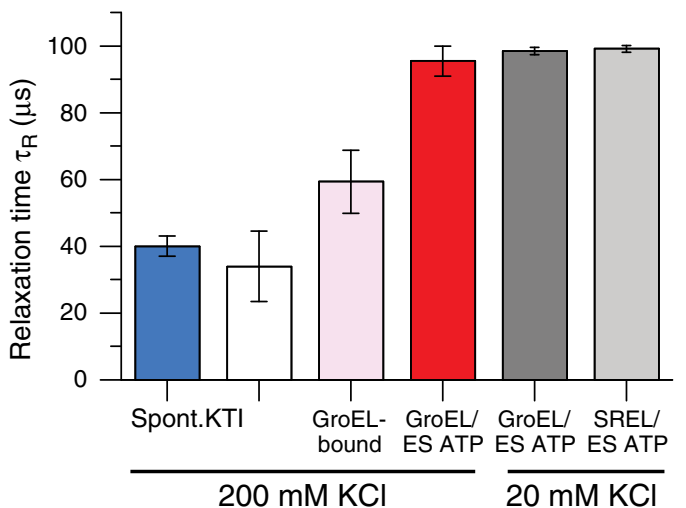

C
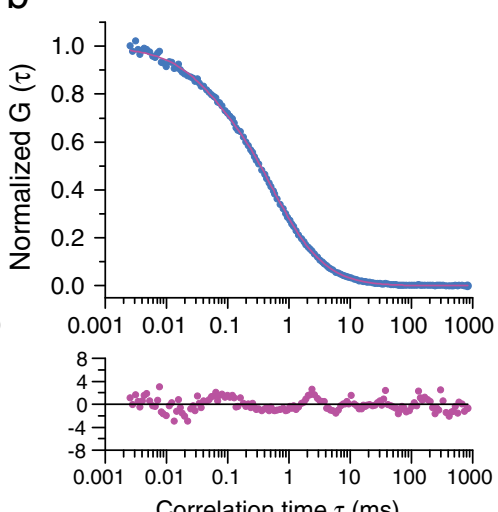

Correlation time $\tau(\mathrm{ms})$
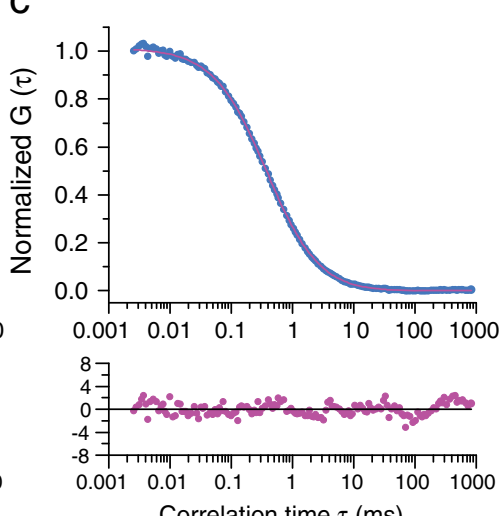

Correlation time $\tau(\mathrm{ms})$

e

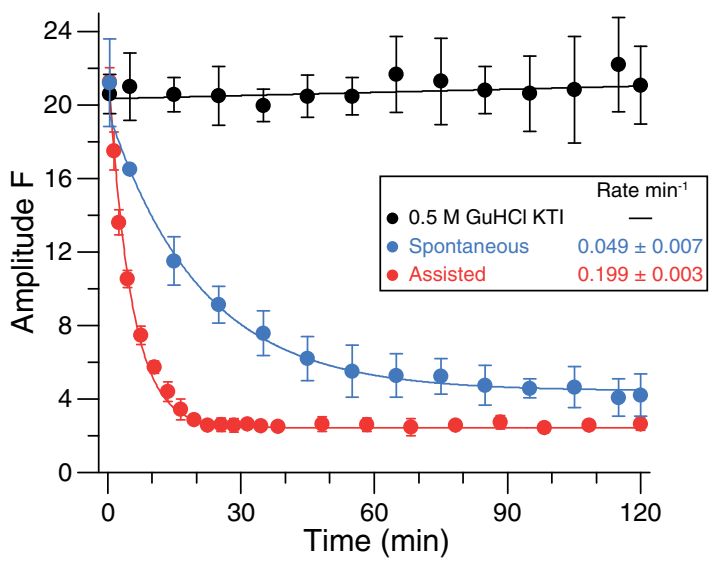


salt buffer condition (Fig. 5f). Thus, the environment of the chaperonin cage causes a considerable reduction of chain entropy, presumably promoting the conversion of dynamic folding intermediate to the native state.

\section{Substrate protein spends most of its time in the encapsulated state during folding}

The finding that, during GroEL/ES cycling, the substrate protein is conformationally restricted to the same extent as upon stable encapsulation in SREL/ ES suggested that the vast majority of folding protein is in the encapsulated state. This would be consistent with recapture of non-native DM-MBP by GroEL occurring in less than $0.3 \mathrm{~s}$ at $25^{\circ} \mathrm{C}$ [22]. Indeed the diffusion time of $\mathrm{DM}-\mathrm{MBP}(\mathrm{DL})$ measured during the first minute of folding with GroEL/ES/ATP was virtually identical with that of the GroEL-bound protein and well discriminated from the fast diffusion time of spontaneously refolding DM-MBP(DL) (Fig. $\mathrm{S} 5 \mathrm{a})$. To quantify the relative amounts of GroEL-bound and encapsulated DM-MBP during the GroEL/ES reaction, we used single-molecule spFRET. DM-MBP(DL) showed similar FRET efficiency distributions when bound to GroEL or SREL with $\sim 34-40 \%$ of molecules being in an expanded state ( $f_{E}$ of 0.06 and 0.1 , respectively) and the remainder populating a broad distribution around an intermediate $f_{\mathrm{E}}$ of $\sim 0.4$ (Fig. $6 \mathrm{a}$ and $\mathrm{b}$, left panels). During the first minute of encapsulation in SREL/ES, essentially all DM-MBP(DL) molecules populated a broad range of collapsed states around an $f_{\mathrm{E}}$ of 0.66 (Fig. 6b, right panel). In contrast, during the first minute of the GroEL/ES reaction, a bimodal $f_{\mathrm{E}}$ distribution was observed, with the low $f_{\mathrm{E}}$ peak representing GroEL-bound molecules and the high $f_{\mathrm{E}}$ population representing encapsulated and folded molecules (Fig. 6a, middle panel). The fraction of folded molecules was $\sim 12 \%$, as determined by addition of apyrase after $1 \mathrm{~min}$ to stop folding and revert the not-yet folded molecules to the bound state with low $f_{\mathrm{E}}$ value (Fig. 6a, right panel). Taking into consideration that the $\sim 12 \%$ of folded $\mathrm{DM}-\mathrm{MBP}(\mathrm{DL})$ were no longer GroEL associated, we calculated that $\sim 18 \%$ of GroEL-associated molecules were bound and $~ 82 \%$ were encapsulated. Thus, during the cycling GroEL/ ES reaction, the vast majority of substrate protein is in the encapsulated state during folding, in agreement with the PET-FCS measurements.

Next, we measured the duration of the GroEL/ES ATPase cycle as a function of substrate concentration. GroEL hydrolyzed ATP at a rate of $\sim 53$ ATP $\min ^{-1}$ at $20{ }^{\circ} \mathrm{C}$ and GroES reduced the rate to $\sim 21$ ATP $\min ^{-1}$ [50] (Fig. S5b). The presence of non-native substrate protein has been reported to stimulate the ATPase [29] by triggering ADP and GroES release from the GroEL trans-ring $[38,51,52]$. A single round of substrate binding and encapsulation occurs in the time it takes GroEL to hydrolyze 7 ATP (the hemi-cycle) in the presence of GroES and non-native substrate. Because spontaneous folding of DM-MBP is slow, we could measure the GroEL ATPase under conditions of substrate excess. At a concentration of $0.2 \mu \mathrm{M}$ GroEL and $0.4 \mu \mathrm{M}$ GroES, the initial ATPase rate reached a maximum of $\sim 59$ ATP per GroEL 14-mer min $^{-1}$ at $~ 0.8 \mu \mathrm{M}$ denatured DM-MBP and remained constant at higher DM-MBP concentrations (Fig. 6c and Fig. S4b). Thus, the duration of the GroEL/ES hemi-cycle at substrate

Fig. 5. Analysis of DM-MBP refolding and conformational dynamics by PET-FCS. (a-c) Normalized fluorescence auto-correlation amplitudes $G(\mathrm{~T})$ for DM-MBP(Atto655) ( $a$ and b) and for Atto655-labeled wild-type MBP(312C) (c) during spontaneous refolding at final concentration of $1 \mathrm{nM}$ in buffer $\mathrm{A}$ at $20^{\circ} \mathrm{C}$. Auto-correlation data collected during the first minute after 200-fold dilution of denatured protein into refolding buffer A (final MBP, $1 \mathrm{nM}$ ) were fitted with a one-diffusion model ( $a$ and $c$ ) or with a one-diffusion one-exponential model (b), which contains an exponential time constant $\left(\mathrm{T}_{\mathrm{R}}\right)$ with an amplitude $F$ in addition to a diffusion component $\left(T_{D}\right.$ and $\rho$ ) (Fig. S3a). Residuals of the fits are shown. Auto-correlation curves shown are representative of least three independent experiments. (d) PET-FCS curves for DM-MBP(Atto655) after completion of spontaneous refolding (open circles) and immediately upon dilution from denaturant into refolding buffer $\mathrm{A}$ (folding intermediate) (filled circles). Auto-correlation curves shown are representative of least three independent experiments. (e) Rates of spontaneous and GroEL/ES-assisted refolding of DM-MBP(Atto655) measured as the time-dependent decrease in PET-FCS amplitude ( $F$ ) (Fig. S3a). FCS recording was started immediately upon initiation of refolding and was continued for $2 \mathrm{~h}$. $F$ was also analyzed upon dilution of denatured DM-MBP(Atto655) into buffer A containing $0.5 \mathrm{M} \mathrm{GuHCl}$ to stabilize the kinetically trapped intermediate (KTI). Time windows of 2 min for early time points and $10 \mathrm{~min}$ for late time points (GroEL/ES-assisted) or time windows of $10 \mathrm{~min}$ for early and late time points (spontaneous refolding and kinetically trapped intermediate) were correlated and fitted as in (b). Refolding rates were extracted by single exponential fits to plots of the amplitude of the exponential component $F$ versus refolding time. Averages \pm SD from at least 3 independent measurements are shown. (f) Conformational dynamics of DM-MBP is decreased inside the chaperonin cage. Relaxation time ( $\mathrm{T}_{\mathrm{R}}$ ) of the PET signal of DM-MBP(Atto655) upon 200-fold dilution from $6 \mathrm{M} \mathrm{GuHCl}$ into refolding buffer $\mathrm{A}$ ( $200 \mathrm{mM} \mathrm{KCl}$ ) (spontaneous refolding; spont.) or into buffer A containing $0.5 \mathrm{M} \mathrm{GuHCl}$ to form $\mathrm{KTI}$ or into buffer A containing GroEL $(2 \mu \mathrm{M})$ (GroEL-bound) or during the first minute of GroEL/ES-assisted refolding in buffer A. $T_{R}$ was also analyzed during the first minute of GroEL/ES- and SREL/ES-assisted refolding of $10 \mathrm{M}$ urea-denatured protein in buffer B $(20 \mathrm{mM} \mathrm{KCl})$. FCS auto-correlation curves were fitted to a one-diffusion one-exponential model and $\mathrm{T}_{\mathrm{R}}$ values were extracted, which are inversely proportional to protein chain motion. Data represent averages \pm SD from at least 3 independent measurements. 

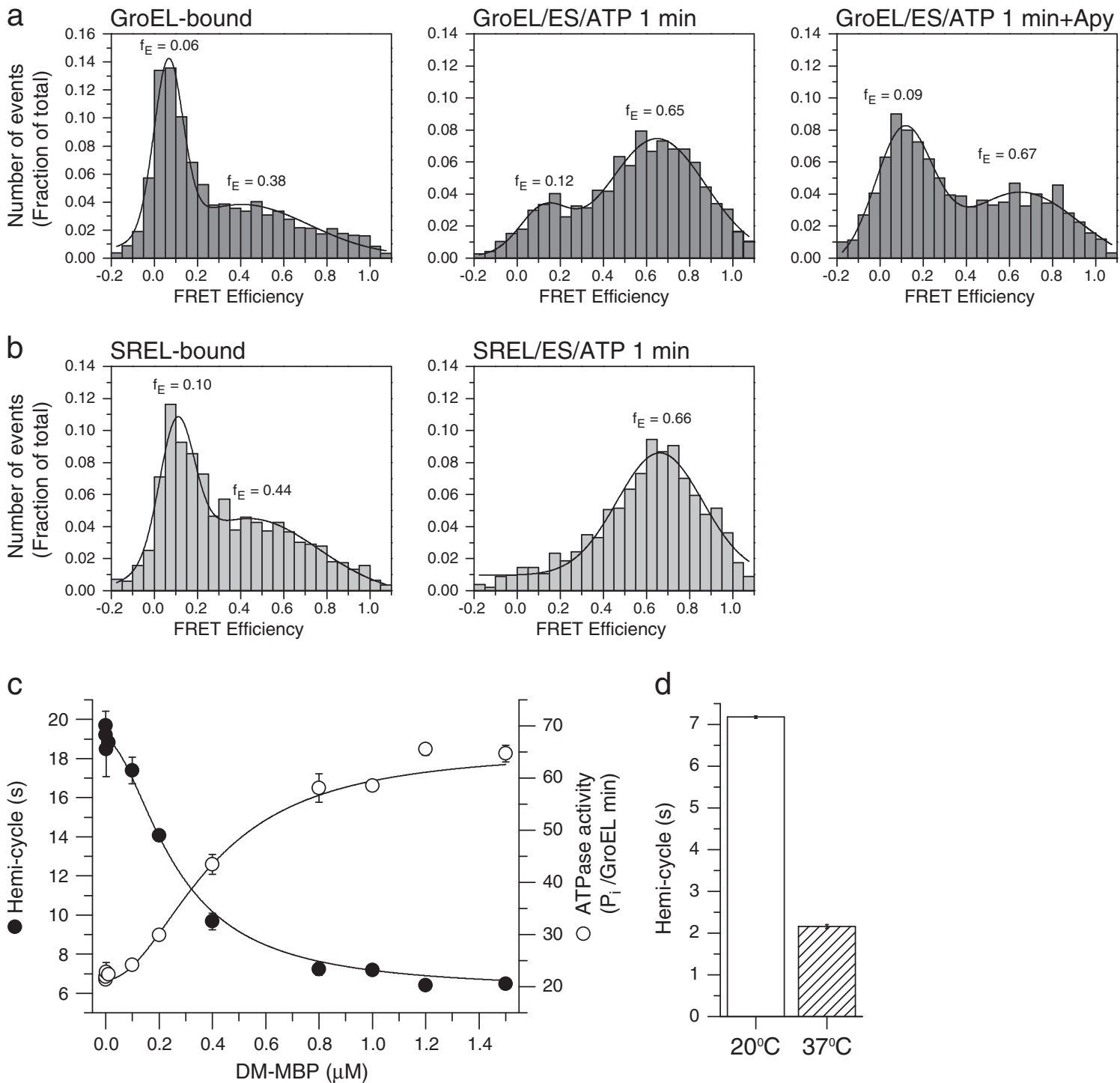

Fig. 6. Analysis of GroEL-bound and encapsulated DM-MBP during assisted refolding. (a) FRET efficiency ( $\left.f_{E}\right)$ histograms from spFRET measurements of GroEL-bound DM-MBP(DL), as well as during the first minute of DM-MBP(DL) refolding and after stopping refolding at $1 \mathrm{~min}$ with apyrase (Apy). Measurements were performed as in Fig. $3 a$ and $\mathrm{d}$. Histograms representative of at least three independent experiments are shown. (b) $f_{\mathrm{E}}$ histograms as in (a) performed for SREL-bound DM-MBP(DL) and during the first minute of DM-MBP(DL) refolding while encapsulated in SREL/ES (buffer B at $20^{\circ} \mathrm{C}$ ). Peak values of a Gaussian fit to the $t_{\mathrm{E}}$ distributions are indicated. Histograms representative of at least three independent experiments are shown. (c) Measuring the GroEL ATPase (open circles) and the duration of the GroEL hemi-cycle (filled circles) in the presence of GroES and increasing concentrations of non-native DM-MBP. Denatured DM-MBP was diluted 200-fold to final concentrations from 0 to $1.5 \mu \mathrm{M}$ into buffer A containing $0.2 \mu \mathrm{M}$ GroEL and $0.4 \mu \mathrm{M}$ GroES. ATPase activities were measured photometrically using a NADH coupled enzymatic assay at $20^{\circ} \mathrm{C}$ (see Materials and Methods). \pm SD from at least 3 independent measurements. (d) Duration of GroEL ATPase hemi-cycle as measured in (c) in the presence of $1 \mu \mathrm{M}$ non-native DM-MBP at $20^{\circ} \mathrm{C}$ and $37^{\circ} \mathrm{C}$. Data represent averages \pm SD from at least 3 independent measurements.

saturation is $\sim 7 \mathrm{~s}$ at $20^{\circ} \mathrm{C}$ (Fig. $6 \mathrm{c}$ and d). As measured by stopped-flow mixing, binding of denatured DM-MBP to GroEL is complete after $\sim 0.3 \mathrm{~s}$ and encapsulation upon addition of ATP and GroES is complete after $\sim 0.5 \mathrm{~s}$ [18]. This would mean that the substrate spends $\sim 1 \mathrm{~s}$ in the GroEL-bound state and $\sim 6 \mathrm{~s}$ in the GroEL/ES cage, corresponding to $\sim 14 \%$ and $\sim 86 \%$ of hemi-cycle duration, respectively, in good agreement with the fraction of bound $(\sim 18 \%)$ and encapsulated substrate ( 82\%) 
determined by spFRET mentioned above. The hemi-cycle duration in the presence of excess substrate at $37^{\circ} \mathrm{C}$ was $\sim 2.2 \mathrm{~s}$ (Fig. $6 \mathrm{~d}$ ). Thus, the reaction has a $Q_{10}$ temperature coefficient of $\sim 2$, consistent with classical Arrhenius behavior and suggesting that all steps of the GroEL/ES mechanism undergo similar temperature-dependent rate acceleration.

\section{Role of the net-negative charged GroEL cis-cavity wall}

The wall of the GroEL cis-cavity has a net charge of minus 42 resulting from a cluster of exposed negatively charged residues (Glu252, Asp253, Glu255, Asp359, Asp361 and Glu363) [17]. Most of these are highly conserved among GroEL homologs but have no apparent function in binding of substrate or GroES. We have previously reported that mutating three of these residues (Asp359, Asp361 and Glu363) in SREL to lysines [SR(KKK2)], converting the cavity net charge to 0 , impairs the ability of SR(KKK2)/ES to fold DM-MBP and bacterial ribulose bisphosphate carboxylase/oxygenase (RuBisCO), but not mitochondrial rhodanese [17]. Both DM-MBP and RuBisCO experience a significant rate acceleration of folding with wild-type SREL/ES [12,17], while rhodanese does not, suggesting that the negative charges have a role in enhancing folding kinetics. Here we analyzed this possibility using the $\mathrm{SR}(\mathrm{KKK} 2)$ and $\mathrm{EL}(\mathrm{KKK} 2)$ mutants.

Under standard conditions of physiological salt concentration, GroEL/ES accelerated the spontaneous folding of DM-MBP(312C) by $\sim 4.5$-fold. In contrast, no rate acceleration was observed with EL(KKK2)/ES (Fig. 7a). Remarkably, EL(KKK2)/ES did not restrict the chain dynamics of DM-MBP(Atto655) during folding as measured by PET-FCS, in striking contrast to GroEL/ES (Fig. 7a). Notably, DM-MBP(DL) when bound to EL(KKK2) displayed the same conformational properties as when bound to GroEL, as demonstrated by spFRET measurements (Fig. S5c). Moreover, during the first minute of folding with EL(KKK2)/ES/ATP, the diffusion time of DM-MBP(DL) was identical with that of the EL(KKK2)-bound protein (Fig. S5a), indicating that essentially all substrate was chaperonin associated. The fraction of bound and encapsulated substrate analyzed from spFRET histograms was $\sim 16 \%$ and $\sim 84 \%$, respectively, close to the values obtained with GroEL/ES (Fig. S4c and Fig. 6a). The ATPase activity of EL(KKK2) was similar to that of GroEL and was inhibited by GroES. However, unlike GroEL, excess non-native DM-MBP had only a minor effect in stimulating the ATPase of EL(KKK2)/ ES (Fig. S5b). These results suggested that the charge properties of the cis-cavity wall may have a dual role in entropically confining encapsulated substrate protein and in coupling the presence of substrate with the ATPase activity of GroEL.

To uncouple these two effects, we next used $\mathrm{SR}(\mathrm{KKK} 2)$ to analyze the chain dynamics of DM-MBP during folding when stably encapsulated. GroES-mediated substrate encapsulation by $\mathrm{SR}(\mathrm{KKK} 2)$ at low salt (Fig. S5d and e) was as efficient as with SREL (Fig. S4). When bound to $\mathrm{SR}(\mathrm{KKK} 2)$, DM-MBP(DL) displayed similar conformational properties as when bound to GroEL or SREL (Fig. S5c and f). During the first minute of encapsulation, DM-MBP(DL) populated compact conformations, as observed with SREL/ES (Fig. S5f). GroEL/ES and SREL/ES mediated the refolding of DM-MBP $(312 \mathrm{C})$ at essentially the same rate (measured at low salt) (Fig. 7b), and folding by
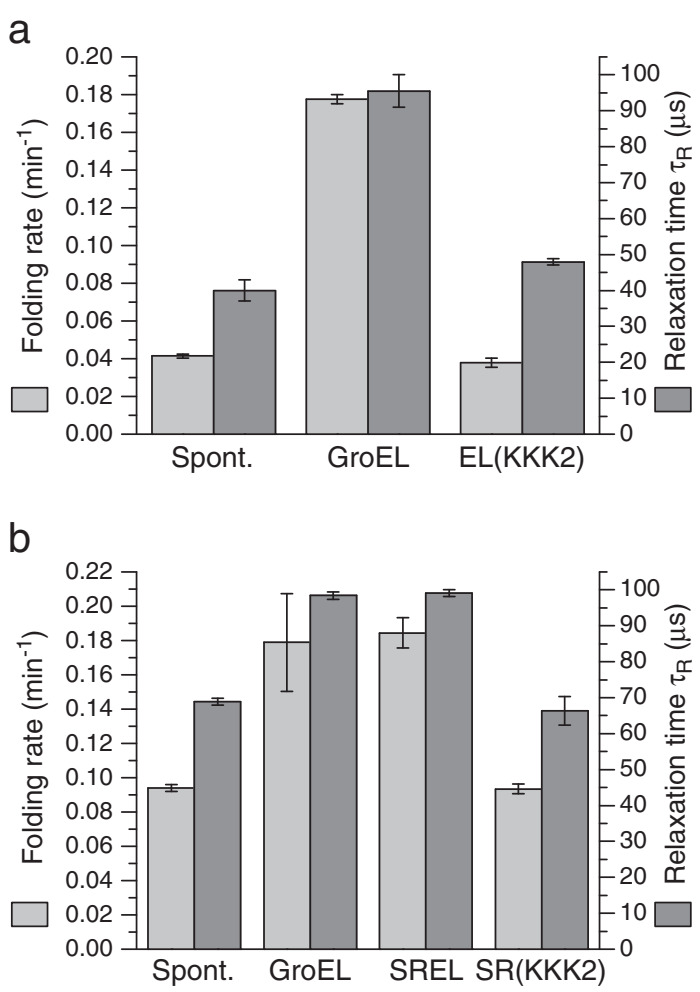

Fig. 7. Effect of net-negative charges in the chaperonin cis-cavity on DM-MBP folding and conformational dynamics. (a) Spontaneous (Spont.), GroEL/ES-assisted and $E L(K K K 2) / E S$-assisted refolding of unlabeled DM-MBP(312C) in buffer A (200 mM KCl) were measured by monitoring the increase in $\operatorname{Trp}$ fluorescence at $20^{\circ} \mathrm{C}$ as described in Fig. S3. Relaxation times $\left(T_{R}\right)$ of the PET signal of DM-MBP(Atto655) were measured during the first minute of spontaneous and assisted refolding. \pm SD from at least 3 independent measurements. (b) Spontaneous and assisted refolding with GroEL/ES, SREL/ES and $\mathrm{SR}(\mathrm{KKK} 2) / \mathrm{ES}$ were measured in buffer $\mathrm{B}(20 \mathrm{mM} \mathrm{KCl})$ and $T_{R}$ values were also analyzed during the first minute of refolding as in (a). Data represent averages $\pm S D$ from at least 3 independent measurements. 
SR(KKK2)/ES was not accelerated beyond the spontaneous rate (Fig. $7 b$ ). Interestingly, while the folding rates with GroEL/ES and SREL/ES are salt independent, the KKK2 mutant displays a similar salt dependence of folding rate as spontaneous renaturation (Fig. 7a and b and Fig. S2) [22]. Compared to high salt, the low salt condition resulted in reduced chain dynamics (slower $\mathrm{T}_{\mathrm{R}}$ ) and $\sim 2$-fold faster folding for the spontaneous renaturation (Fig. $7 a$ and b). Accordingly, in low salt, folding in SR(KKK2)/ES is $\sim 2$-fold faster than in EL(KKK2)/ES at high salt (Fig. 7a and b). Importantly, DM-MBP(Atto655) when inside SR(KKK2)/ES nevertheless displayed significantly higher chain dynamics ( $T_{R}$ of $66 \pm 4 \mu \mathrm{s}$ ) compared to SREL/ES ( $T_{R}$ of $99 \pm 1 \mu \mathrm{s}$ ) (Fig. 7b). Together these findings indicate that the net-negative charge of the cis-cavity plays a critical role in entropically confining dynamic folding intermediates of encapsulated substrate, thereby accelerating their conversion to the native state.

\section{Discussion}

\section{Active versus passive GroEL/ES function}

It has been argued that accelerated folding of DM-MBP by GroEL/ES $[17,19,22]$ is due to the ability of chaperonin to prevent reversible aggregation that would otherwise reduce the rate, but not the yield, of spontaneous folding $[24,26]$. The basic assumption of this passive cage model is that GroEL/ES functions solely as an anti-aggregation device and that folding inside the GroEL/ES cage occurs at the same rate as it would during spontaneous folding at infinite dilution [25]. Here we have tested this idea by monitoring folding by single-molecule experiments at $100 \mathrm{pM}$ DM-MBP. Since the probability of more than one molecule of DM-MBP residing in the confocal observation volume is $\leq 1 \%$ at this concentration, we were able to compare the rates of GroEL/ES-assisted folding and spontaneous folding at de facto infinite dilution. The absence of aggregation during spontaneous folding was confirmed by FCS and dcFCCS measurements. Using GroEL to sort non-native from native molecules, we showed by spFRET and FCS that GroEL/ES accelerates fluorescent-labeled DM-MBP refolding 4- to 8-fold, demonstrating the active chaperonin mechanism.

\section{Active cage model versus iterative annealing}

According to the iterative annealing model, substrate binding and release during ATP-dependent GroEL/ES cycling serve to unfold kinetically trapped folding intermediates, affording them a chance at productive folding either inside or outside the GroEL/ ES cage $[27,44]$. Using SREL/ES, we demonstrated that a single round of encapsulation inside the chaperonin cage is sufficient to achieve accelerated DM-MBP folding at full yield. This argues for encapsulation being the active principle. However, an active cage may function synergistically with iterative annealing for certain substrates, for example, when a fraction of molecules were to form long-lived, misfolded states during assisted folding. We also note that the SREL experiments do not rule out the possible contribution of a single round of unfolding to subsequent accelerated folding inside the cage. A conformational expansion of substrate protein upon binding to GroEL and additional stretching upon ATP-mediated apical domain movement has been observed [18,34,35] (as well as this study) (Fig. 1b). Furthermore, during GroES-mediated encapsulation, the protein is released in a step-wise fashion, with the less hydrophobic and thus less tightly bound, sequence elements dissociating before the more hydrophobic ones [18]. This mode of release would modulate the mechanism of hydrophobic collapse and may contribute to avoiding the formation of kinetically trapped folding intermediates.

The iterative annealing model assigns no specific function to the chaperonin cage in aggregation prevention or accelerating folding and accordingly suggests that folding may equally occur inside the GroEL/ES cage or outside [27] (Fig. 1b). Out of cage-folding would require that not-yet folded protein spends significant time in free solution during GroEL/ ES cycling. This is inconsistent with our PET-FCS and spFRET measurements, which demonstrate that, in the cycling reaction, non-native DM-MBP spends $\sim 80 \%$ of the duration of the GroEL/ES hemi-cycle (7 ATP hydrolyzed; $7 \mathrm{~s}$ at $20^{\circ} \mathrm{C}$ ) in the encapsulated state and the remainder bound to the apical GroEL domains. Considering that GroEL recaptures non-native DM-MBP from solution within $0.3 \mathrm{~s}$ or less (at $25^{\circ} \mathrm{C}$ ) [18], the fraction of out of cage folding would be insignificant $(<5 \%)$. Moreover, even vastly increasing the concentration of GroEL/ ES relative to substrate, as in our single-molecule experiments (100 pM DM-MBP, $2 \mu \mathrm{M}$ GroEL/4 $\mu \mathrm{M}$ GroES), did not slow folding kinetics or yield, which can only be explained by in cage folding. It has also been argued that, at $37^{\circ} \mathrm{C}$, the dwell time of substrate inside the GroEL/ES cage becomes too short for efficient folding [27]. We measured the duration of the GroEL/ES hemi-cycle under substrate saturation at $37^{\circ} \mathrm{C}$ to be $2.2 \mathrm{~s}$. Making the reasonable assumption that all the steps of the GroEL/ES reaction undergo temperature-dependent acceleration, the vast majority of folding would nevertheless occur in cage. Indeed, authentic GroEL-dependent proteins are typically highly aggregation prone in a temperature-dependent manner $[16,53,54]$. Under so-called non-permissive conditions, where irreversible aggregation prohibits spontaneous folding, the GroEL/ES-assisted folding of these proteins stops immediately when rapid recapture of 
folding intermediate by GroEL is prevented [12], indicating that folding must occur in cage.

\section{Function of the GroEL/ES cage}

Protein encapsulation by chaperonin has been proposed to serve a dual purpose: it prevents aggregation and accelerates folding for certain proteins, adjusting folding speed relative to the rate of protein synthesis and thereby preventing the accumulation of unfolded or misfolded protein molecules [12]. Rate enhancement of folding was attributed to an effect of steric confinement, entropically destabilizing dynamic folding intermediates and thereby facilitating their conversion to the native state $[17,19,22]$. Two physical properties of the GroEL/ES cage may be critical in this regard: the volume of the cage relative to the size of the folding protein (i.e., steric confinement proper $[17,19,41,42,55])$ and the negative net charge of the cavity wall $[17,19]$ that has been suggested to increase the hydrophobic effect by ordering water structure [56]. In the present study, we used PET-FCS to directly measure the effect of encapsulation on polypeptide chain dynamics. While binding of unfolded protein to GroEL restricts chain dynamics only moderately, we find that encapsulation results in a marked restriction of flexibility, as reflected in an increase of the $T_{R}$ of the DM-MBP folding intermediate from $40 \pm 3 \mu$ s during spontaneous refolding to $99 \pm 1 \mu$ s when encapsulated. Notably, $T_{R}$ of the folding intermediate was very similar when measured during active GroEL/ES cycling or upon stable encapsulation in SREL/ES. This provides direct evidence that, during folding, the protein spends the vast majority of the time in the encapsulated state. Contrary to a recent report [57], our experiments with SREL/ES did not reveal a functionally significant "escape" of DM-MBP from the non-cycling SREL/ES cage.

Using EL(KKK2) or SR(KKK2), a mutant in which the negative net charge of the cavity wall of 42 is reduced to 0 , we analyzed the effect of cavity charge on substrate chain dynamics by PET-FCS. Strikingly, these measurements showed that the KKK2 cage is unable to restrict the conformational motion of encapsulated protein relative to the folding intermediate in free solution. This correlates with a loss of function of the KKK2 mutant in accelerating DM-MBP folding. Thus, in essence, removal of the negative net charge converts the active GroEL/ES cage to a passive cage. Moreover, we found that the negatively charged residues exposed in the cis-cavity are required for the stimulation of the GroEL ATPase by substrate protein. It thus appears that the cage senses the encapsulated protein. In summary, we suggest that steric confinement and the negatively charged cavity wall function cooperatively in promoting folding.

\section{Materials and Methods}

\author{
Strains, plasmids and proteins
}

The E. colistrains DH5a and BL21 (DE3) Gold (Stratagene) were used for cloning and protein expression, respectively. DM-MBP (MBP V8G, Y283D) and its cysteine variants, DM-MBP(312C) and DM-MBP(30C/312C), were constructed in $\mathrm{pCH}$ vectors ( $\mathrm{T} 7$ promoter) by site-directed mutagenesis using QuikChange (Stratagene) and were expressed, purified and labeled with fluorophore $[17,18]$ (see Supplementary Methods for details).

GroEL, GroES, SREL, EL(KKK2) and SR(KKK2) were expressed and purified as previously described [17,19]. GroEL preparations were subjected to rigorous quality control by the following assays: ATPase activity in presence and absence of GroES [19], rhodanese aggregation prevention [58] and DM-MBP refolding [17]. GroES preparations were controlled for efficient inhibition of GroEL ATPase activity and ability to accelerate DM-MBP refolding.

\section{DM-MBP refolding}

DM-MBP was unfolded in $6 \mathrm{M} \mathrm{GuHCl} / 10 \mathrm{mM}$ DTT or in $10 \mathrm{M}$ urea/10 $\mathrm{mM}$ DTT for $1 \mathrm{~h}$ at $50{ }^{\circ} \mathrm{C}$, as indicated in the figure legends. Spontaneous refolding was initiated upon 200 -fold dilution into refolding buffer $\mathrm{A}$ [20 mM Tris- $\mathrm{HCl}$ (pH 7.5), $200 \mathrm{mM} \mathrm{KCl}$ and $\left.5 \mathrm{mM} \mathrm{Mg}\left(\mathrm{C}_{2} \mathrm{H}_{3} \mathrm{O}_{2}\right)_{2}\right]$ or refolding buffer $\mathrm{B}$ [50 mM Hepes- $\mathrm{NaOH}(\mathrm{pH} 7.5), 20 \mathrm{mM} \mathrm{KCl}$ and $10 \mathrm{mM} \mathrm{MgCl}_{2}$ ] as indicated in the figure legends. For assisted refolding, denatured DM-MBP was diluted 200 -fold into refolding buffer containing either $2 \mu \mathrm{M}$ GroEL or $1 \mu \mathrm{M}$ SREL and refolding was initiated upon addition of $4 \mu \mathrm{M}$ GroES and $5 \mathrm{mM}$ ATP.

In ensemble experiments (100 nM DM-MBP), folding was recorded by the time-dependent increase in the intrinsic Trp fluorescence (excitation: $295 \mathrm{~nm}$; emission: $345 \mathrm{~nm}$ ) of DM-MBP and its variants on a Fluorolog F3-22 spectrofluorometer (Horiba), equipped with Peletier thermostat set to $20^{\circ} \mathrm{C}$ (GroEL and GroES do not contain Trp) [17]. Photobleaching was carefully avoided by limiting the excitation slit width to $2 \mathrm{~nm}$, with the emission slit width being set to $8 \mathrm{~nm}$. Fluorescence signal was excited and emission was collected every $30 \mathrm{~s}$ for a 100-ms window using an automated shutter. Note that, at low concentrations of DM-MBP, bleaching of Trp fluorescence may result in measuring seemingly faster folding rates than at higher concentrations.

For DM-MBP(DL), refolding could not be measured by Trp fluorescence due to quenching. However, fluorescence of the donor dye in the folding intermediate was significantly lower than in the native state of DM-MBP(DL). The time-dependent increase in donor fluorescence was used as a measure of DM-MBP(DL) folding in ensemble measurements at $100 \mathrm{nM}$ (excitation wavelength, $532 \mathrm{~nm}$; emission wavelength, $550 \mathrm{~nm}$ ).

\section{Analysis of protein encapsulation}

Encapsulation experiments were performed in buffer $B$. DM-MBP(Atto655) was unfolded in $10 \mathrm{M}$ urea/10 mM DTT 
for $1 \mathrm{~h}$ at $50^{\circ} \mathrm{C}$. The denatured protein was diluted 200 -fold (30 $\mathrm{nM}$ final) into refolding buffer containing $1 \mu \mathrm{M}$ SREL or $\mathrm{SR}(\mathrm{KKK} 2)$. The reaction was incubated for $5 \mathrm{~min}$ at room temperature. Refolding was started by addition of $3 \mu \mathrm{M}$ GroES and $1 \mathrm{mM}$ ATP at $20^{\circ} \mathrm{C}$. The reaction was separated on a Superdex 200 PC3.2/30 gel-filtration column (Amersham Biosciences), equilibrated in buffer B/50 mM urea/1 mM ATP, either immediately or after $30-60 \mathrm{~min}$ of incubation at $20^{\circ} \mathrm{C}$ or after $30-60$ min of incubation at $20^{\circ} \mathrm{C}$ and dissociation of the SREL/ES complex by the addition of $50 \mathrm{mM}$ CDTA/70 mM $\mathrm{GuHCl} / 200 \mathrm{mM} \mathrm{KCl}$. Fractions were collected and analyzed by $15 \%$ SDS-PAGE, Coomassie staining and fluorescence imaging (FujiFilm FLA3000) and were quantified by densitometry.

\section{ATPase assay}

The ATPase activity of $0.2 \mu \mathrm{M}$ GroEL or EL(KKK2) was measured in buffer $A$ at $20^{\circ} \mathrm{C}$ in the absence or in the presence of $0.4 \mu \mathrm{M}$ GroES or $0.4 \mu \mathrm{M}$ GroES with increasing concentration of denatured DM-MBP (diluted 200 -fold from $6 \mathrm{M} \mathrm{GuHCl}$ ). Control reactions received equivalent amounts of $\mathrm{GuHCl}$ ( $30 \mathrm{mM}$ final). The hydrolysis of ATP to ADP was followed photometrically using an $\mathrm{NADH}$ coupled enzymatic assay (2 $\mathrm{mM}$ phosphoenolpyruvate, $30 / 20 \mathrm{U} \mathrm{ml}^{-1}$ pyruvate kinase/lactate dehydrogenase, $0.5 \mathrm{mM} \mathrm{NADH}$ and $1 \mathrm{mM} \mathrm{ATP}$ ) at $20^{\circ} \mathrm{C}$ in a spectrophotometer (Jasco) essentially as previously described [59].

\section{Single-molecule spectroscopy}

Single-molecule spectroscopy was performed on a Microtime 200 inverse time-resolved fluorescence microscope (PicoQuant) using pulsed interleaved excitation [60] (see Supplementary Methods for details). The instrument was maintained at a constant temperature of $20^{\circ} \mathrm{C}$. dcFCCS and FCS were used to investigate the oligomeric state of DM-MBP during refolding [18,22], while spFRET and FCS were used to assess folding rates of DM-MBP at $100 \mathrm{pM}$. The significant size difference of folded DM-MBP monomer ( 41 kDa) and non-native DM-MBP in complex with GroEL $(\sim 830 \mathrm{kDa})$ results in different diffusion rates $\left(106 \pm 6 \mu \mathrm{m}^{2} \mathrm{~s}^{-1}\right.$ and $49 \pm$ $1 \mu \mathrm{m}^{2} \mathrm{~s}^{-1}$, respectively) that can be monitored using FCS. The auto-correlation data were fitted with the following one triplet one-diffusion equation using the Symphotime software (PicoQuant):

$$
\begin{aligned}
G(T)= & {\left[1-T+T \times e^{\left(\frac{T}{T_{\mathrm{T}}}\right)}\right] } \\
& \times\left[\rho \times\left(1+\frac{T}{T_{\mathrm{D}}}\right)^{-1} \times\left(1+\frac{T}{T_{\mathrm{D}} \times K^{2}}\right)^{-1 / 2}\right]
\end{aligned}
$$

The mean diffusion time $T_{D}$ of particles through the focal spot is described by the structural parameter $\mathrm{K}=z_{0} / \omega_{0}$ where $z_{0}$ and $\omega_{0}$ denote the axial and radial dimensions of the confocal volume, respectively. The amplitude of the correlation function is denoted by $\rho$. The first term is used to compensate for fast dynamics arising from dye photophysics such as triplet blinking with the amplitude $T$ on the timescale $\mathrm{T}_{\mathrm{T}}$ [61]. Diffusion coefficients were calculated using the following equation

$D=\frac{\left(V_{\text {eff }} \times \pi^{-3 / 2} \times K^{-1}\right)^{2 / 3}}{4 \times T_{\mathrm{D}}}$

by calibrating the confocal volume $V_{\text {eff }}$ with Atto655 dye, for which accurate diffusion parameters have been published [62]. To analyze refolding kinetics, we plotted the mean diffusion time, reflecting a shift of DM-MBP molecules from GroEL bound to free against the refolding time and fitted it with a single exponential rate.

PET-FCS was used as an approach to assess conformational dynamics of refolding DM-MBP molecules $[47,48]$. DM-MBP(Atto655) (or Atto655-labeled wild-type MBP) was unfolded in $6 \mathrm{M} \mathrm{GuHCl} / 10 \mathrm{mM}$ DTT for $1 \mathrm{~h}$ at $20{ }^{\circ} \mathrm{C}$ or $10 \mathrm{M}$ urea/10 mM DTT for $1 \mathrm{~h}$ at $50{ }^{\circ} \mathrm{C}$. Refolding was started by 200 -fold dilution of the protein (final $100 \mathrm{pM}$ to $1 \mu \mathrm{M}$ ) into refolding buffer A or buffer B as indicated in the figure legends. FCS measurements were performed immediately. In order to resolve fast dynamics in the microsecond timescale, we recorded fluorescence on two detectors simultaneously. Cross-correlation of the signals allowed removal of detector after pulsing. The correlated data were fitted with either a single component diffusion model

$G(\tau)=\rho \times\left(1+\frac{T}{T_{\mathrm{D}}}\right)^{-1} \times\left(1+\frac{T}{T_{\mathrm{D}} \times K^{2}}\right)^{-1 / 2}$

or the following one-exponential one-diffusion equation, with the exponential term describing amplitude $F$ and relaxation time $T_{R}$ of $P E T$

$$
\begin{aligned}
G(T)= & {\left[1-F+F \times e^{\left(-\frac{T}{T_{\mathrm{R}}}\right)}\right] } \\
& \times\left[\rho \times\left(1+\frac{T}{T_{\mathrm{D}}}\right)^{-1} \times\left(1+\frac{T}{T_{\mathrm{D}} \times K^{2}}\right)^{-1 / 2}\right]
\end{aligned}
$$

in Symphotime (PicoQuant). For relaxation time extraction, only the first or the last $30 \mathrm{~s}$ of a $2-\mathrm{h}$ refolding experiment was considered. For folding rate extraction, the measurement was subdivided into 2 min time windows and extracted values for $F$ were plotted against refolding time. These data were fitted with a single exponential function in Origin (OriginLabs) to give refolding rates.

\section{Acknowledgements}

We gratefully acknowledge the expert technical assistance by N. Wischnewski and A. R. Lange.

Author Contributions: A.J.G. and S.H. designed and performed the experiments and wrote the first draft of the manuscript; G.M. performed the analyses of protein encapsulation. F.U.H. and M.H.H. advised and supervised the project and wrote the paper.

Conflict of Interest Statement: The authors declare that they have no conflict of interest. 


\section{Appendix A. Supplementary data}

Supplementary data include Supplementary Methods and five figures. Supplementary data associated with this article can be found, in the online version, at http://dx.doi.org/10.1016/j.jmb.2014.04. 018.

Received 22 February 2014; Received in revised form 16 April 2014; Accepted 21 April 2014

Available online 6 May 2014

Keywords:
chaperonins;
GroEL;
GroES;
DM-MBP;

$\uparrow$ A.J.G. and S.H. contributed equally to this work, and they share first authorship.

Abbreviations used: DM-MBP, double-mutant maltose binding protein; spFRET, single-pair fluorescence resonance energy transfer; dcFCCS, dual-color fluorescence crosscorrelation spectroscopy; FCS, fluorescence correlation spectroscopy; PET, photoinduced electron transfer.

\section{References}

[1] Frydman J. Folding of newly translated proteins in vivo: the role of molecular chaperones. Annu Rev Biochem 2001;70:603-47.

[2] Fenton WA, Horwich AL. Chaperonin-mediated protein folding: fate of substrate polypeptide. Q Rev Biophys 2003;36:229-56.

[3] Gershenson A, Gierasch LM. Protein folding in the cell: challenges and progress. Curr Opin Struct Biol 2011;21:32-41.

[4] Saibil HR, Fenton WA, Clare DK, Horwich AL. Structure and allostery of the chaperonin GroEL. J Mol Biol 2013;425:1476-87.

[5] Kim YE, Hipp MS, Bracher A, Hayer-Hartl M, Hartl FU. Molecular chaperone functions in protein folding and proteostasis. Annu Rev Biochem 2013;82:323-55.

[6] Vitlin Gruber A, Nisemblat S, Azem A, Weiss C. The complexity of chloroplast chaperonins. Trends Plant Sci 2013;18:688-94.

[7] Mayhew M, Da Silva ACR, Martin J, Erdjument-Bromage H, Tempst $P$, Hartl FU. Protein folding in the central cavity of the GroEL-GroES chaperonin complex. Nature 1996;379:420-6.

[8] Weissman JS, Rye HS, Fenton WA, Beechem JM, Horwich AL. Characterization of the active intermediate of a GroELGroES-mediated protein folding reaction. Cell 1996;84:481-90.

[9] Hayer-Hartl MK, Weber F, Hartl FU. Mechanism of chaperonin action: GroES binding and release can drive GroELmediated protein folding in the absence of ATP hydrolysis. EMBO J 1996;15:6111-21.
[10] Xu ZH, Horwich AL, Sigler PB. The crystal structure of the asymmetric GroEL-GroES-(ADP)7 chaperonin complex. Nature 1997;388:741-9.

[11] Rye HS, Burston SG, Fenton WA, Beechem JM, Xu Z, Sigler $\mathrm{PB}$, et al. Distinct actions of cis and trans ATP within the double ring of the chaperonin GroEL. Nature 1997;388:792-8.

[12] Brinker A, Pfeifer G, Kerner MJ, Naylor DJ, Hartl FU, HayerHartl M. Dual function of protein confinement in chaperoninassisted protein folding. Cell 2001;107:223-33.

[13] Wang JD, Herman C, Tipton KA, Gross CA, Weissman JS. Directed evolution of substrate-optimized GroEL/S chaperonins. Cell 2002;111:1027-39.

[14] Meyer AS, Gillespie JR, Walther D, Millet IS, Doniach S, Frydman J. Closing the folding chamber of the eukaryotic chaperonin requires the transition state of ATP hydrolysis. Cell 2003;113:369-81.

[15] Figueiredo L, Klunker D, Ang D, Naylor DJ, Kerner MJ, Georgopoulos C, et al. Functional characterization of an archaeal GroEL/GroES chaperonin system-significance of substrate encapsulation. J Biol Chem 2004;279:1090-9.

[16] Kerner MJ, Naylor DJ, Ishihama Y, Maier T, Chang HC, Stines $\mathrm{AP}$, et al. Proteome-wide analysis of chaperonin-dependent protein folding in Escherichia coli. Cell 2005;122:209-20.

[17] Tang YC, Chang HC, Roeben A, Wischnewski D, Wischnewski N, Kerner MJ, et al. Structural features of the GroEL-GroES nano-cage required for rapid folding of encapsulated protein. Cell 2006;125:903-14.

[18] Sharma S, Chakraborty K, Mueller BK, Astola N, Tang YC, Lamb $\mathrm{DC}$, et al. Monitoring protein conformation along the pathway of chaperonin-assisted folding. Cell 2008;133:142-53.

[19] Tang YC, Chang HC, Chakraborty K, Hartl FU, Hayer-Hartl $M$. Essential role of the chaperonin folding compartment in vivo. EMBO J 2008;27:1458-68.

[20] Clare DK, Bakkes PJ, van Heerikhuizen $H$, van der Vies SM, Saibil HR. Chaperonin complex with a newly folded protein encapsulated in the folding chamber. Nature 2009;457:107-13.

[21] Zhang J, Baker ML, Schroder GF, Douglas NR, Reissmann $\mathrm{S}$, Jakana J, et al. Mechanism of folding chamber closure in a group II chaperonin. Nature 2010;463:379-83.

[22] Chakraborty K, Chatila M, Sinha J, Shi Q, Poschner BC, Sikor $\mathrm{M}$, et al. Chaperonin-catalyzed rescue of kinetically trapped states in protein folding. Cell 2010;142:112-22.

[23] Chen D-H, Madan D, Weaver J, Lin Z, Schroder GF, Chiu W, et al. Visualizing GroEL/ES in the act of encapsulating a folding protein. Cell 2013;153:1354-65.

[24] Apetri AC, Horwich AL. Chaperonin chamber accelerates protein folding through passive action of preventing aggregation. Proc Natl Acad Sci USA 2008;105:17351-5.

[25] Horwich AL, Apetri AC, Fenton WA. The GroEL/GroES cis cavity as a passive anti-aggregation device. FEBS Lett 2009;583:2654-62.

[26] Tyagi NK, Fenton WA, Deniz AA, Horwich AL. Double mutant MBP refolds at same rate in free solution as inside the GroEL/ GroES chaperonin chamber when aggregation in free solution is prevented. FEBS Lett 2011;585:1969-72.

[27] Yang D, Ye X, Lorimer GH. Symmetric GroEL:GroES2 complexes are the protein-folding functional form of the chaperonin nanomachine. Proc Natl Acad Sci USA 2013;110:E4298-305.

[28] Braig K, Otwinowski Z, Hegde R, Boisvert DC, Joachimiak A, Horwich AL, et al. The crystal structure of the bacterial chaperonin GroEL at 2.8 Å. Nature 1994;371:578-86. 
[29] Martin J, Langer T, Boteva R, Schramel A, Horwich AL, Hartl FU. Chaperonin-mediated protein folding at the surface of GroEL through a "molten globule"-like intermediate. Nature 1991;352:36-42.

[30] Fenton WA, Kashi Y, Furtak K, Horwich AL. Residues in chaperonin GroEL required for polypeptide binding and release. Nature 1994;371:614-9.

[31] Hayer-Hartl MK, Ewbank JJ, Creighton TE, Hartl FU. Conformational specificity of the chaperonin GroEL for the compact folding intermediates of alpha-lactalbumin. EMBO $J$ 1994;13:3192-202.

[32] Horovitz A, Willison KR. Allosteric regulation of chaperonins. Curr Opin Struct Biol 2005;15:646-51.

[33] Lin Z, Rye HS. Expansion and compression of a protein folding intermediate by GroEL. Mol Cell 2004;16:23-34.

[34] Lin Z, Madan D, Rye HS. GroEL stimulates protein folding through forced unfolding. Nat Struct Mol Biol 2008;15:303-11.

[35] Kim SY, Miller EJ, Frydman J, Moerner WE. Action of the chaperonin GroEL/ES on a non-native substrate observed with single-molecule FRET. J Mol Biol 2010;401:553-63.

[36] Lin Z, Puchalla J, Shoup D, Rye HS. Repetitive protein unfolding by the trans ring of the GroEL-GroES chaperonin complex stimulates folding. J Biol Chem 2013;288:30944-55.

[37] Takei $Y$, lizuka $R$, Ueno T, Funatsu T. Single-molecule observation of protein folding in symmetric GroEL-(GroES)2 complexes. J Biol Chem 2012;287:41118-25.

[38] Ye X, Lorimer GH. Substrate protein switches groE chaperonins from asymmetric to symmetric cycling by catalyzing nucleotide exchange. Proc Natl Acad Sci USA 2013;110: E4289-97.

[39] Ellis RJ. Molecular chaperones. Opening and closing the Anfinsen cage. Curr Biol 1994;4:633-5.

[40] Ellis RJ, Hartl FU. Protein folding in the cell: competing models of chaperonin function. FASEB J 1996;10:20-6.

[41] Baumketner A, Jewett A, Shea JE. Effects of confinement in chaperonin assisted protein folding: rate enhancement by decreasing the roughness of the folding energy landscape. $J$ Mol Biol 2003;332:701-13.

[42] Hayer-Hartl M, Minton AP. A simple semiempirical model for the effect of molecular confinement upon the rate of protein folding. Biochemistry 2006;45:13356-60.

[43] Lucent D, Vishal V, Pande VS. Protein folding under confinement: a role for solvent. Proc Natl Acad Sci USA 2007;104:10430-4.

[44] Thirumalai D, Lorimer GH. Chaperonin-mediated protein folding. Annu Rev Biophys Biomol Struct 2001;30:245-69.

[45] Sparrer H, Lilie H, Buchner J. Dynamics of the GroEL protein complex: effects of nucleotides and folding mutants. J Mol Biol 1996;258:74-87.

[46] Chun SY, Strobel S, Bassford P, Randall LL. Folding of maltose-binding protein. Evidence for the identity of the rate- determining step in vivo and in vitro. $\mathrm{J}$ Biol Chem 1993;268:20855-62.

[47] Neuweiler H, Johnson CM, Fersht AR. Direct observation of ultrafast folding and denatured state dynamics in single protein molecules. Proc Natl Acad Sci USA 2009;106:18575-80.

[48] Sauer M, Neuweiler H. PET-FCS: probing rapid structural fluctuations of proteins and nucleic acids by single-molecule fluorescence quenching. Methods Mol Biol 2014;1076:597-615.

[49] Motojima F, Motojima-Miyazaki Y, Yoshida M. Revisiting the contribution of negative charges on the chaperonin cage wall to the acceleration of protein folding. Proc Natl Acad Sci USA 2012;109:15740-5.

[50] Chandrasekhar GN, Tilly K, Woolford C, Hendrix R, Georgopoulos C. Purification and properties of the GroES morphogenetic protein of Escherichia coli. J Biol Chem 1986;261:12414-9.

[51] Martin J, Mayhew M, Langer T, Hartl FU. The reaction cycle of GroEL and GroES in chaperonin-assisted protein folding. Nature 1993;366:228-33.

[52] Hayer-Hartl MK, Martin J, Hartl FU. Asymmetrical interaction of GroEL and GroES in the ATPase cycle of assisted protein folding. Science 1995;269:836-41.

[53] Fujiwara K, Ishihama Y, Nakahigashi K, Soga T, Taguchi H. A systematic survey of in vivo obligate chaperonin-dependent substrates. EMBO J 2010;29:1552-64.

[54] Calloni G, Chen T, Schermann SM, Chang H-C, Genevaux $\mathrm{P}$, Agostini $\mathrm{F}$, et al. DnaK functions as a central hub in the $E$. coli chaperone network. Cell Rep 2012;1:251-64.

[55] Sirur A, Best RB. Effects of interactions with the GroEL cavity on protein folding rates. Biophys J 2013;104:1098-106.

[56] England JL, Lucent D, Pande VS. A role for confined water in chaperonin function. J Am Chem Soc 2008;130:11838-9.

[57] Motojima F, Yoshida M. Polypeptide in the chaperonin cage partly protrudes out and then folds inside or escapes outside. EMBO J 2010;29:4008-19.

[58] Weber F, Hayer-Hartl M. Prevention of rhodanese aggregation by the chaperonin GroEL. In: Schneider C, editor. Chaperonin Protocols: Methods in Molecular Biology, 140. Totowa, NJ: Humana Press; 2000. p. 111-5.

[59] Poso D, Clarke AR, Burston SG. A kinetic analysis of the nucleotide-induced allosteric transitions in a single-ring mutant of GroEL. J Mol Biol 2004;338:969-77.

[60] Muller BK, Zaychikov E, Brauchle C, Lamb DC. Pulsed interleaved excitation. Biophys J 2005;89:3508-22.

[61] Widengren J, Mets U, Rigler R. Fluorescence correlation spectroscopy of triplet states in solution: a theoretical and experimental study. J Phys Chem 1995;99:13368-79.

[62] Müller CB, Loman A, Pacheco V, Koberling F, Willbold D, Richtering $W$, et al. Precise measurement of diffusion by multi-color dual-focus fluorescence correlation spectroscopy. Europhys Lett 2008;83:46001. 